\title{
Coupling in-situ X-ray micro- and nano-tomography and discrete element method for investigating high temperature sintering of metal and ceramic powders
}

\author{
Zilin Yan ${ }^{1}$, Christophe L. Martin ${ }^{1, \star}$, Didier Bouvard ${ }^{1, \star \star}$ David Jauffrès $^{1}$, Pierre Lhuissier ${ }^{1}$, Luc Salvo ${ }^{1}$, Luis Olmos $^{2}$, \\ Julie Villanova ${ }^{3}$, and Olivier Guillon ${ }^{4}$ \\ ${ }^{1}$ Univ. Grenoble Alpes, CNRS, SIMAP, 38000 Grenoble, France \\ ${ }^{2}$ Universidad Michoacana de San Nicolás de Hidalgo, IIMM and INICIT, Fco. J. Mujica S/N, Ed. C-2 C.U., Morelia, Michoacán, \\ C.P. 58060, Mexico \\ ${ }^{3}$ ESRF The European Synchrotron, CS 40220, 38043 Grenoble Cedex 9, France \\ ${ }^{4}$ Forschungszentrum Jülich Institute of Energy and Climate Research, D-52425 Jülich, Germany
}

\begin{abstract}
The behaviour of various powder systems during high temperature sintering has been investigated by coupling X-ray microtomography and discrete element method (DEM). Both methods are particularly relevant to analyse particle interactions and porosity changes occurring during sintering. Two examples are presented. The first one deals with a copper powder including artificially created pores which sintering has been observed in situ at the European synchrotron and simulated by DEM. 3D images with a resolution of $1.5 \mu \mathrm{m}$ have been taken at various times of the sintering cycle. The comparison of the real displacement of particle centers with the displacement derived from the mean field assumption demonstrates significant particle rearrangement in some regions of the sample. Although DEM simulation showed less rearrangement, it has been able to accurately predict the densification kinetics. The second example concerns multilayer ceramic capacitors (MLCCs) composed of hundreds of alternated metal electrode and ceramic dielectric layers. The observation of Ni-based MLCCs by synchrotron nanotomography at Argon National Laboratory with a spatial resolution between 10 and $50 \mathrm{~nm}$ allowed understanding the origin of heterogeneities formed in Ni layers during sintering. DEM simulations confirmed this analysis and provided clues for reducing these defects.
\end{abstract}

\section{Introduction}

$\mathrm{X}$-ray microtomography is a non-destructive method used to observe the microstructure of materials in 3D [1]. This technique is particularly powerful for particulate materials where the pore phase is transparent to X-rays. Although technologically challenging, in situ observation of a material during a high temperature thermal treatment, as for example powder sintering, is particularly attractive. With third generation synchrotrons, typical resolution is $1 \mu \mathrm{m}$ and typical scanning time is a few tens of seconds, which is convenient for analysing the dynamics of the sintering of metal powders. More recently, novel nano-tomography configurations allow the antagonism between high resolution (sub-micronic) and rapid scan times (tens of seconds) to be resolved. This opens new avenues for the observation of micronic ceramic particles during sintering. Discrete Element Methods (DEM) are relevant for modelling powder sintering since they explicitly take into account the particulate nature of the material. DEM models for sintering should include the specific contact forces that mimic interparticle bonding at high temperature. The writing of

\footnotetext{
${ }^{\star}$ e-mail: christophe.martin@grenoble-inp.fr

$\star \star$ e-mail: didier.bouvard@grenoble-inp.fr
}

these contact force includes surface energy and diffusion coefficients. The overall problem must be treated as the minimization of the total surface energy of the packing. A difficulty of DEM modelling is the availability of appropriate data for both feeding and validating simulations. In situ X-ray tomography is clearly appropriate for this purpose as it can provide quantitative data on particle assembly dynamics during sintering. Here, we report on two examples of coupling synchrotron tomography experiments and discrete element simulations.

\section{DEM model}

The details of the DEM model may be found in [2]. The main ingredients, specific to the high temperature sintering problem are recalled here. Particles are modeled as spheres that may indent each other due to surface energy minimization. Parhami and McMeeking' model, which assumes that grain boundary and surface diffusions are the major mechanisms of mass transport, is used [3]. The normal contact force between two sintering particles writes:

$$
N_{s}=\frac{\pi a_{s}^{4}}{8 \Delta_{b}} \frac{\mathrm{d} \delta}{\mathrm{d} t}-\pi \gamma_{s}\left[8 R^{*}\left(1-\cos \frac{\psi}{2}\right)+a_{s} \sin \frac{\psi}{2}\right]
$$



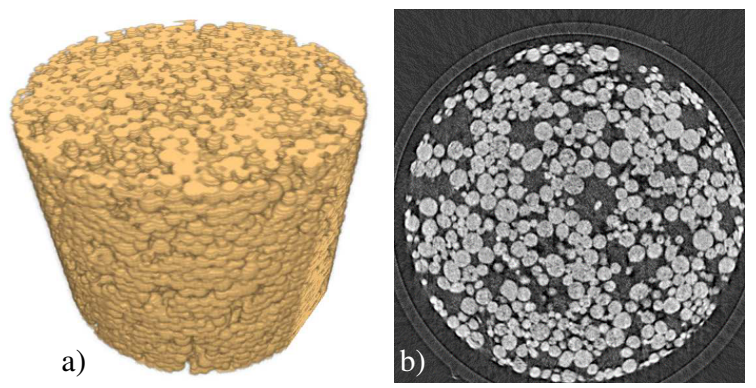

Figure 1. 3D rendering (a) and virtual slice (b) of the image of a $1 \mathrm{~mm}$ diameter copper sample with artificially created pores.

where $a_{s}$ is the contact radius, $\delta$ is the indentation, $\Delta_{b}$ is a temperature dependent diffusion coefficient, $\gamma_{s}$ is the surface energy, $R^{*}$ is the effective radius of the two particles and $\psi$ is the dihedral angle that defines the equilibrium shape of the contact. The first term on the rhs of eqn (1) is the normal viscous term while the second term, which is tensile, is the sintering term. A tangential force, opposes the tangential relative velocity at the contact $\left(\frac{\mathrm{d} u}{\mathrm{~d} u}\right)$ :

$$
T_{s}=-\eta \frac{\pi a_{s}^{2} R^{* 2}}{2 \Delta_{b}} \frac{\mathrm{d} u}{\mathrm{~d} t}
$$

where $\eta$ is a is a viscous parameter with no dimension. Because of material transport, the contact radius $a_{s}$ writes:

$$
a_{s}=4 R^{*} \delta
$$

which differs by a factor 4 from its equivalent in elasticity.

No external load is applied on the particle packing and particles motion is only dictated by surface minimization $\left(\gamma_{s}\right.$ term in eqn (1)).

\section{Copper based powders}

Experiments were carried out at the European Synchrotron in Grenoble with copper based powder systems sintered up to $1060^{\circ} \mathrm{C}$ in a furnace located between the X-ray source and the detector. 3D images with a resolution of $1.5 \mu \mathrm{m}$ and a scanning time of $1 \mathrm{~min}$ were recorded at various stages of the thermal cycle [4]. Among the various investigated systems, we focus on the one with artificially created pores (so-called porous), which mimics materials with large, controlled porosity as filters, SOFC electrodes, bone implants [5]. Copper powder with 0-63 $\mu \mathrm{m}$ size was first mixed with 10 vol.\% of $\mathrm{NaCl}$ salt particles with a diameter between 40 and $63 \mu \mathrm{m}$ as pore formers. This mixture was then poured in a $1 \mathrm{~mm}$ diameter quartz capillary and pre-sintered at $500^{\circ} \mathrm{C}$ during 30 minutes. The capillary was removed after cooling and the salt was dissolved by keeping the sample in distilled water for 5 hours. Fig. 1 shows a $3 \mathrm{D}$ rendering and a virtual slice of an image of the sample. Sintering was achieved at $1050^{\circ} \mathrm{C}$ under reducing atmosphere ( $\mathrm{He}-5$ at.\% $\mathrm{H} 2)$ to prevent particle oxidation.

The raw 3D images were converted to binary images by thresholding and particle segmentation was achieved
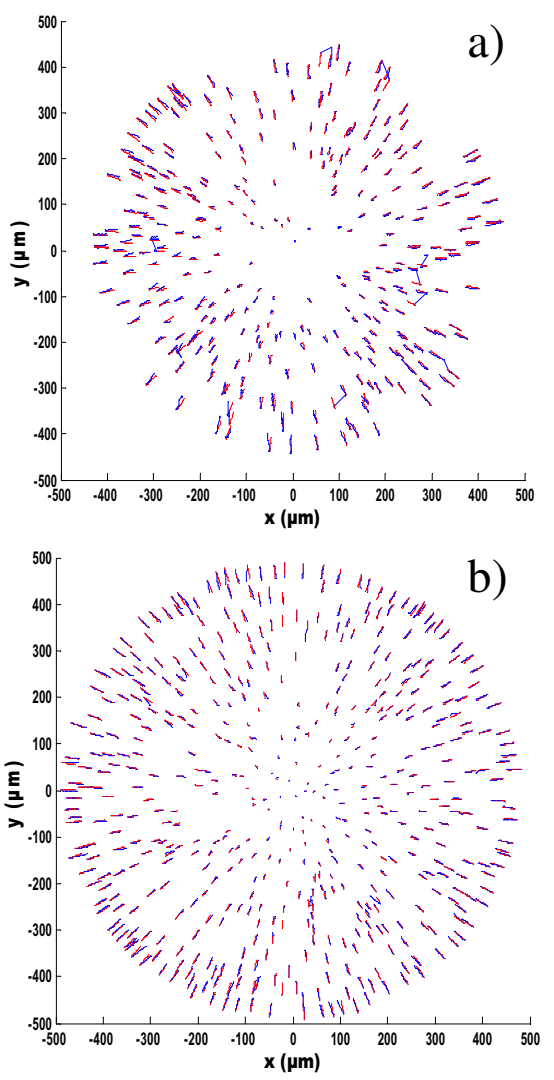

Figure 2. Particle centre trajectories during sintering (in blue) compared with estimated mean field trajectories (in red). a) from microtomography images, b) from DEM simulation.

by a watershed procedure. Fig. 2a compares the displacement of each particle center projected in a horizontal plane with the one predicted by the mean field hypothesis which assumes uniform isotropic shrinkage. The real displacement roughly follows the mean field assumption with significant deviations in a few regions that evidence particle rearrangement.

In parallel, DEM simulations of the sintering have been performed. Building a realistic DEM packing directly from a $3 \mathrm{D}$ image was not satisfactory because of the non-regular particle shape. A customary fabrication method, involving the real particle size distribution of particles and pore formers as well as the initial density of the material to be modeled, which have been deduced from microtomography data, was thus worked out [6]. As shown in Fig 2, the results of porous sample simulation are more consistent with the mean field assumption than the microtomography results. Fig. 3 shows experimental and numerical densification kinetics of both a regular copper sample and the porous sample. The diffusion coefficient has been fitted to get the best agreement between experimental and simulation curves for the regular material. Then, with the same coefficient the sintering of the porous sample was also nicely predicted, which suggests that local rearrangement does not play a significant role in the densification process. 


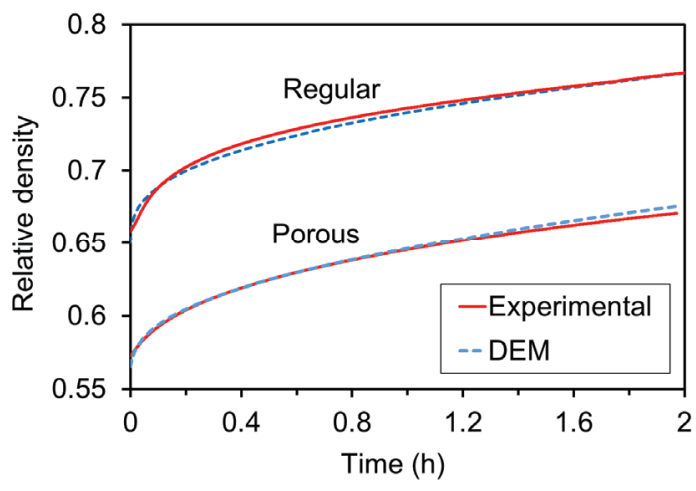

Figure 3. Comparison of experimental and numerical densification kinetics of porous copper sample during sintering.

\section{Multilayer ceramic capacitors}

Multilayer ceramic capacitors (MLCCs) are composed of hundreds of alternated metal electrode and ceramic dielectric layers. Several trillions of them are fabricated each year for electronic parts. Up-to-date MLLCs comprise typically $1 \mu \mathrm{m}$ thick layers made of nano-sized powders. Using the synchrotron radiation provided at Argon National Laboratory, non-sintered (green) and sintered MLCCs composed of Ni electrode and BaTiO3 (BT) dielectric layers have been observed with a spatial resolution between 10 and $50 \mathrm{~nm}$ [8]. The green parts were first machined into $60 \mu \mathrm{m}$ cone shape with a special drilling machine (Fig. 4a). The tip was then milled by dual-beam FIB-SEM into a $20 \mu \mathrm{m}$-diameter cylinder of $20 \mu \mathrm{m}$ height (Fig. 4b). Fig. 4c-f show ex-situ 2D projections and 3D images before and after sintering. The bright areas correspond to the Ni phase while dark ones correspond to the BT phase. Reverse colour code is used in Fig. 5, which shows the in-situ evolution of an MLCC during sintering. Temperature calibrating was carried out by using nanogold particles, which melting was monitored in-situ.

Both post-mortem and in-situ observations indicate that the particulate nature of the layers dictates the microstructural evolution [7]. This is particularly true of the Ni layer, which only contains a few submicronic particles along its thickness. The discontinuities observed in the $\mathrm{Ni}$ layers were found to originate from initial heterogeneities of nickel powders (Fig. 6). The heterogeneity grows into a full defect because of the constraint imposed by the two layers that sandwich the Ni layer. These ceramic layers do not sinter since their sintering temperature is higher. DEM simulations were carried out to refine this scenario.

Particle size and size distribution measured by tomography were used as inputs for simulations. We have observed that the free sintering of a $\mathrm{Ni}$ monolayer leads to a final homogeneous microstructure. When the metal Ni layer is sandwiched between two non-sintering BT layers (Fig. 7), discontinuities appear, thus demonstrating that a constraint is necessary for their nucleation.

Fig. 7 indicates that particles that form clusters in the final microstructure were initially closely packed. As sintering proceeds, local sintering shrinkage mismatch in-

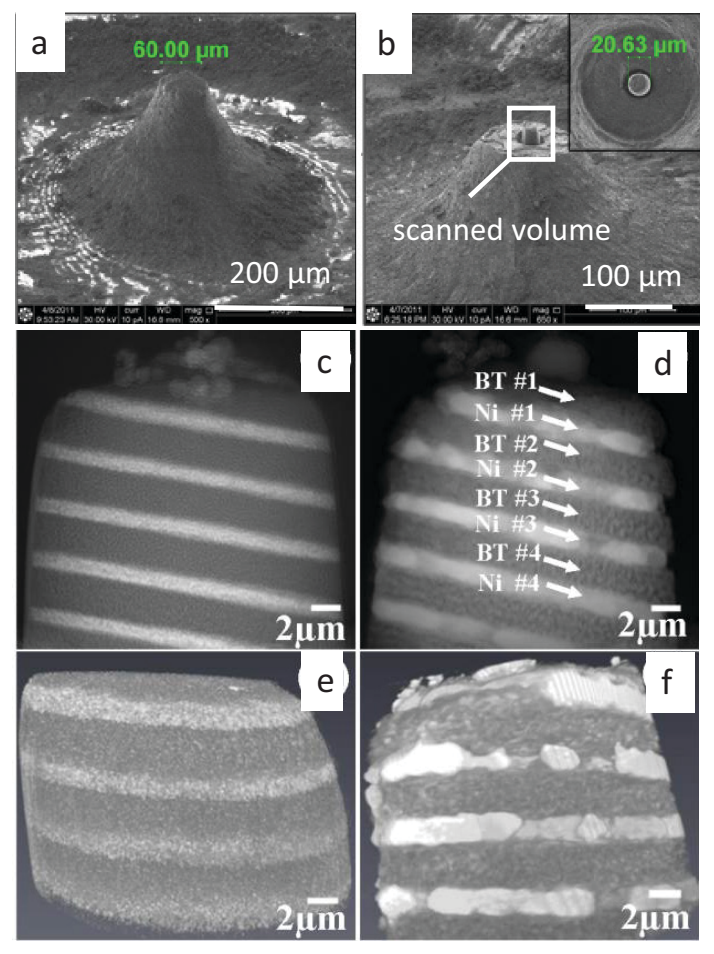

Figure 4. (a-b) MLCC sample preparation for x-ray tomography; 2D projection images (c) before and (d) after sintering, (d), reconstructed 3D microstructure (e) before and (f) after sintering.

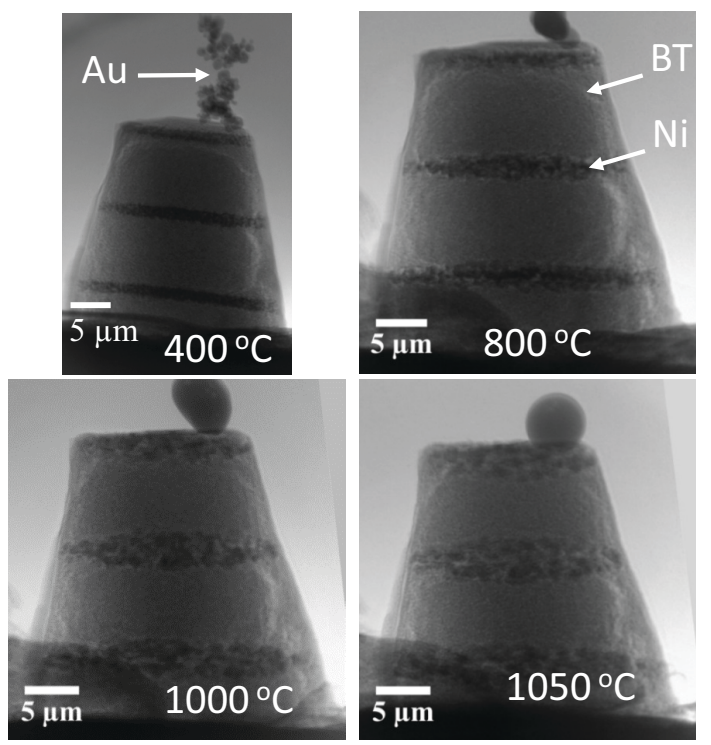

Figure 5. In-situ observation of MLCC sintering at increasing temperature. Au particles are used both for image alignment and for calibrating the furnace temperature.

duces some initially contacting particles to detach from each other. In particular, inspection of eqn (1) shows that small particles sinter faster than large ones. Indeed, we have observed that contacts between large particles were more prone to 'de-sintering' than contacts between small ones. Regions with initially low coordination number and with some large particles at their boundaries detach, re- 

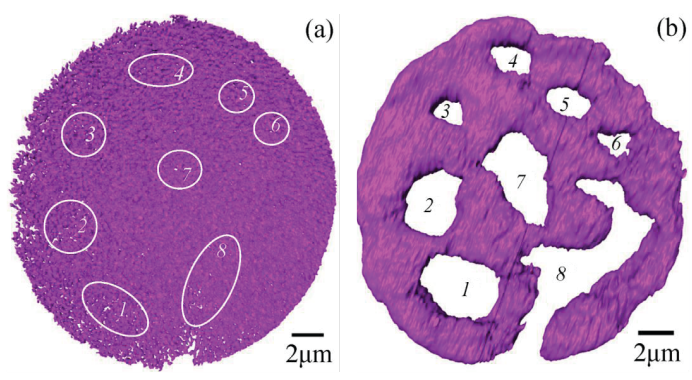

Figure 6. Nickel layer evolution with sintering. Low packing density regions lead to large discontinuities after sintering.

sulting in pore growth. Conversely, regions with high coordination number sinter rapidly leading locally to pore shrinkage. Although particle coarsening is not included in the model (the mechanism that makes large particles grow by eating away smaller ones), it provides a sound scenario for explaining the evolution of the real microstructure pictured in Fig. 6a to the one in Fig. 6b.

The DEM simulations presented here may also be used to propose potential improvements by varying some of the process parameters [8]. We observed that increasing the $\mathrm{Ni}$ layer thickness had a beneficial effect on the discontinuity level because it decreases the constraint that non-sintering layers. However, this is not a option for the MLCC industry, which wants to pack more dielectric layers per unit volume, not less. A more viable route is to decrease the sintering driving force for Ni particles until both the ceramic (BT) and the metal (Ni) layers are at a sufficiently high temperature to sinter at similar rates. In that case, the Ni layer has formed only a small amount of discontinuities when the constraint imposed by the non-sintering BT layer is relieved. In that regard, increasing the heating rate to reach the sintering temperature $\left(1150^{\circ} \mathrm{C}\right)$ should be effective since it reduces the constrained sintering time of the Ni layer. DEM simulations indeed show that increasing the heating rate from $5 \mathrm{~K} / \mathrm{min}$ to $50 \mathrm{~K} / \mathrm{min}$ decreased discontinuity from 6 to $1 \%$. Another option is to use BT as additives in the Ni layer. This has the desired effect of retarding the sintering of the metal layer in the temperature range where the BT layer does not sinter. The addition of 10 vol. \% of BTO nano-particles in the Ni layer decreases the amount of discontinuity from 6 to $4 \%$ at $5 \mathrm{~K} / \mathrm{min}$.

\section{Conclusion}

Both study cases show the interest of coupling microtomography and discrete element modelling to better understand and describe the sintering behaviour of metal and ceramic powders. The occurrence of particle arrangement during sintering copper material with artificially created pores has been assessed. DEM simulation proved that this rearrangement does not play a key role in densification. Concerning multilayer ceramic capacitors, nanotomography observation allowed understanding the origin of defects created during sintering. DEM simulation confirmed this analysis and provided clues for reducing this damage. The versatility of the experimental configuration described here is of great interest for investigations of powders where visualization of thermally activated phenomena is necessary at a fine scale. Current progresses to improve further the acquisition parameters toward even smaller resolution $(<100 \mathrm{~nm})$ together with smaller scan times $(<1 \mathrm{~s})$ will certainly qualify in-situ tomography as a method of choice for small powders at room and high temperature.

\section{References}

[1] L. Salvo, P. Cloetens, E. Maire, S. Zabler, J.J. Blandin, J.Y. Buffière, W. Ludwig, E. Boller, D. Bellet, C. Josserond, Nuclear Instruments and Methods in Physics Research, Section B: Beam Interactions with Materials and Atoms 200, 273 (2003)

[2] Z. Yan, C.L. Martin, O. Guillon, D. Bouvard, Scripta Mater. 69, 327 (2013)

[3] F. Parhami, R.M. McMeeking, Mech. Mater. 27, 111 (1998)

[4] A. Vagnon, J.P. Riviere, J.M. Missiaen, D. Bellet, M.D. Michiel, C. Josserond, D. Bouvard, Acta Mater. 56, 1084 (2008)

[5] L. Olmos, T. Takahashi, D. Bouvard, C.L. Martin, L. Salvo, D. Bellet, M.D. Michiel, Philosophical Magazine 89, 2949 (2009)

[6] L. Olmos, C.L. Martin, D. Bouvard, D. Bellet, M.D. Michielz, J. Am. Ceram. Soc. 92, 1492 (2009)

[7] Z. Yan, O. Guillon, C.L. Martin, S. Wang, C.S. Lee, D. Bouvard, Appl. Phys. Lett. 102, 223107 (2013)

[8] Z. Yan, C.L. Martin, O. Guillon, D. Bouvard, C. Lee, Journal of the European Ceramic Society 34, 3167 (2014)

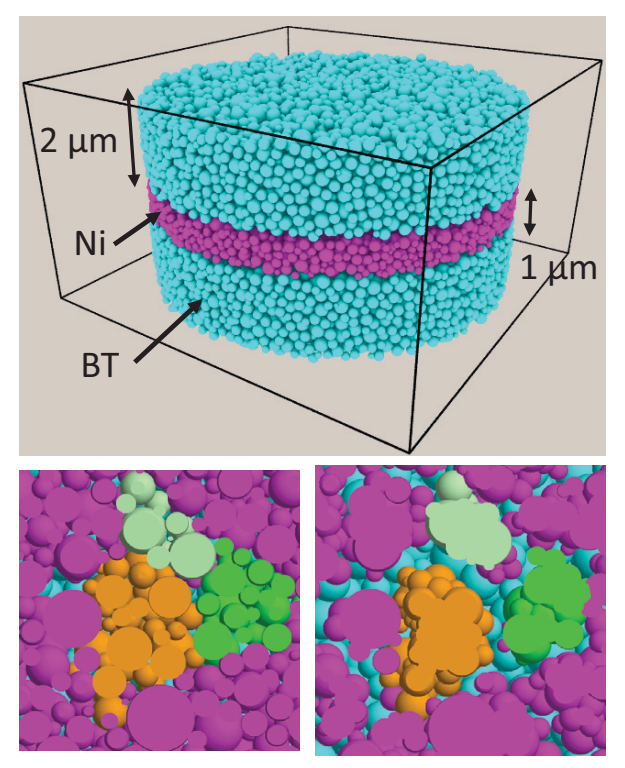

Figure 7. Nickel layer evolution with sintering. Low packing density regions lead to large discontinuities after sintering. 